\title{
Allocation of flow to plots in pressurized irrigation distribution networks
}

\author{
J. Monserrat \\ Department of Agroforestry Engineering, University of Lleida, Spain
}

\begin{abstract}
The method of allocating flow to plots proposed by Clément and Galand (1979) has been revised. Mention is made of its drawbacks owing to the lack of consideration of the specific technical-economic factors of current pressurised irrigation systems (drip or sprinkler) in the plot. A method for fixed irrigation systems is proposed which, based on economic considerations, determines an optimum block area. Bearing in mind the method of irrigation in the plot, an allocation of constant flow is proposed up to a value of maximum surface area, and, from there on, a linear increase related to the plot area. A formula is also presented for calculating the maximum number of blocks from variables easily obtainable in the project phase.
\end{abstract}

Keywords: irrigation network, pressurized irrigation, allocation of flow.

\section{Introduction}

In the current context of high competition for water, there is a need for systems to allow an adequate control of this resource combined with easy management. A good solution is the use of pressurised irrigation distribution systems.

When engineers face the design of a distribution network, they start with different size plots that have to be supplied. The design problem consists of responding to the question of which flow to supply depending on the area of the plot. Once this question is solved, in the case of networks organised by demand, the probability of opening is calculated, and hence, the maximum flows in the network sections, for a specific frequency can be obtained through Clément's first formula $[1,3]$. The following step is the calculation of the diameters of the sections. 
Thus, the problem that concerns us is the allocation of flows to the plots. Being at the early phase of the process this has important repercussions on the final solution. Moreover, this allocation has a direct influence on the degree of satisfaction among the users.

This article is a revision of the Clément and Galand method [2], which is still used in some projects today, and a proposal for a new methodology for allocating supply discharges to the plots. The underlying idea of the method presented is to consider users' requirements, thus planning the network from the bottom up.

The basic equation that has to be fulfilled in an irrigation system is that the volume applied per unit of area, also called irrigation depth or irrigation dose (term on the left of eqn. 1), must be equal to the needs between irrigations (term on the right). The variables in this equation are not homogeneous, but, as they have the same units on both sides, the conversion factor is the same and thus is annulled.

$$
q_{r g} t_{r g}=q_{f b} I_{r} 24
$$

where:

$q_{r g}$ : Irrigation specific discharge, is the flow applied by the planned irrigation system per unit of area, in the case of sprinkler irrigation, it is equivalent to the average rainfall $\left(1 \mathrm{~s}^{-1} \mathrm{ha}^{-1}\right)$.

$t_{r g}$ : Application time, is the irrigation time to apply the depth (h).

$q_{f b}$ : Continuous gross specific discharge. It expresses the needs plus the losses in form of flow per unit of area $\left(l \mathrm{~s}^{-1} \mathrm{ha}^{-1}\right)$

$I_{r}$ : Irrigation interval, that is, the time between two irrigation (days).

The maximum discharge that could be supplied to a plot of a total area $A_{p}$ is:

$$
d_{\text {max }}=q_{r g} A_{p}
$$

That is the flow that could be applied by the irrigation system.

The minimum discharge that could be supplied to a plot of a total area $A_{p}$ is:

$$
d_{\text {min }}=q_{f b} A_{p}
$$

That is the flow to provide the needs.

Any supply discharge between these two could be assigned. Thus the allocation of the supply discharge in the plot is a problem that has no single solution but rather admits multiple proposals. However, as shown below, if various determining factors are taken into account, the optimum solution is fairly limited.

\section{Clément and Galand's method}

Clément and Galand [2] proposed the calculation of a supply specific discharge $\left(q_{p}, 1 \mathrm{~s}^{-1} \mathrm{ha}^{-1}\right)$ which is obtained by dividing the volume required during the 
irrigation interval by the available operating time of the hydrant in the plot $\left(t_{p}\right)$ in the irrigation interval.

Another characteristic of the method is to set standard values for the discharges in the hydrant $(2.78,5.56 \mathrm{l} / \mathrm{s}$...) conditioned by commercial availability on the market. Minimum and maximum plot areas are obtained for each standardised discharge by dividing the discharge by $1.2 q_{p}$ and $0.8 q_{p}$ respectively. That means leaving a range of variation of $\pm 20 \%$ for $q_{p}$. The result of this methodology is shown in figure 1 .

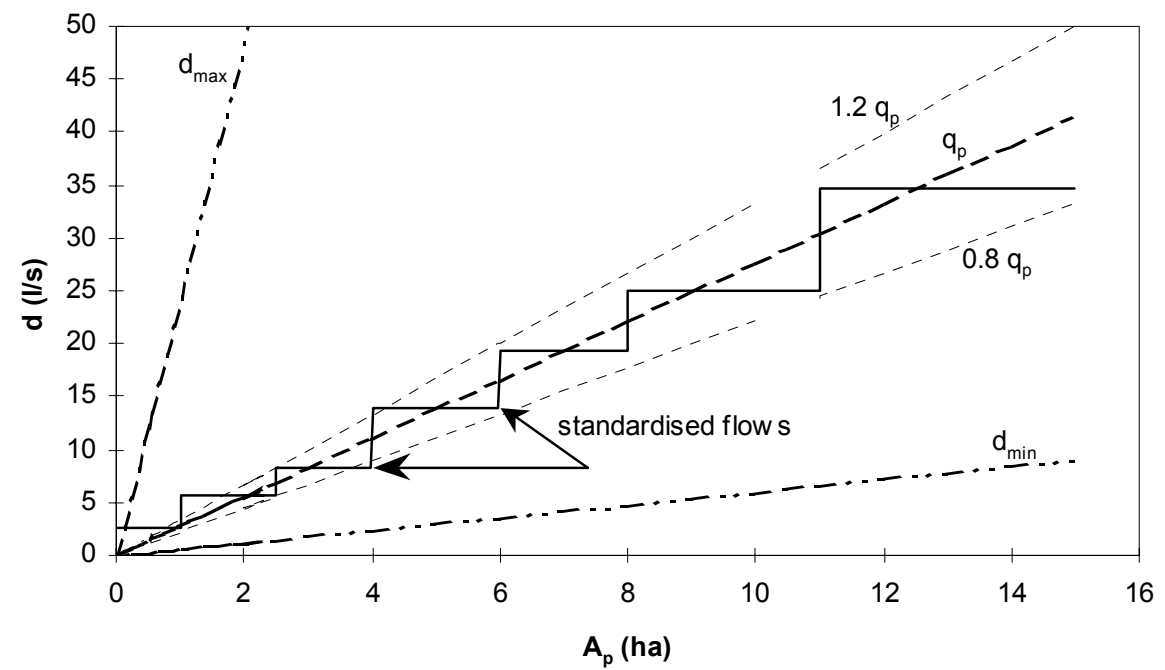

Figure 1: Allocation of discharge depending on plot area proposed by Clément and Galand.

Discussion of this methodology is presented below. The area that each farmer can irrigate simultaneously (Area of the block, $\mathrm{S}_{\mathrm{b}}$ ) is the quotient between the supply discharge $(d)$ and the discharge consumed by the irrigation method per unit of area $\left(q_{r g}\right)$. Thus, given that $q_{r g}$ is constant, the area of the block $\mathrm{S}_{\mathrm{b}}$ grows when $d$ grows. This could be seen comparing figures 5 and 6 , in which the steps in the Clement line are wider for larger plots (fig 6) than for smaller plots (fig 5). The increase in the size of the block with the area of the plot is not logical given that, as shown below, the size of the block relies more on economic factors than the size of the plot.

Another fact to emphasise is the high number of blocks that forces small plots to be made. For example in a plot of 1 ha (Fig. 5), 11 blocks must be made, which is not practical. A possible explanation for these results is that when this method was proposed, the systems were lateral hand-moved sprinkler irrigation and in this case the term block is equivalent to a lateral position, and thus an increase in the number of blocks meant that there were more changes of position but as there was time available between irrigations this was feasible. Nowadays, this technique has 
been abandoned in many countries owing to its high labour requirements and there is a tendency towards buried fixed systems. In this case, a valve is needed for each block, which means a very high cost. Moreover, nowadays the tendency is to irrigate at shorter intervals to increase the hydric comfort of the plant, which is not possible with moveable piping systems. For all these reasons, Clément and Galand's method does not seem recommendable for fixed systems.

We also believe that Clément and Galand's proposal to set standard flows $(2.78,5.56 \mathrm{l} / \mathrm{s})$ was justified by the technology then available (calibrated orifices). However, current hydrant technology based on flow limit and pressure reducing hydraulic valves need not be conditioned by any specific flow as the limiters can be preset to any specified discharge.

\section{Proposed method}

The proposed method could be applied to fixed irrigation systems for both sprinkler and drip irrigation.

As mentioned above, when allocating the discharge to a plot, it is possible to provide the $d_{\max }$ (fig. 1) in such a way that the whole plot can be irrigated simultaneously. Given that this solution maximises the costs of the piping, it is not advisable, whether analysed at a network or plot level.

It is worth asking whether it is necessary to irrigate a plot simultaneously, or whether it can be divided into blocks (block is understood as the area of land irrigated at one time). When the block is very big could be divided into subunits, each of them with a pressure control valve), as a reduction in the simultaneous irrigated area means a reduction in discharge and thus of the diameters of the system.

A first question that arises when subdividing a plot into blocks is the optimum size of the block $\left(A_{b}\right.$ opt $)$. Given that the division into blocks responds to economic criteria, the variation in the unit cost of the block $(€ /$ ha) depending on its size can be analysed. Figure 2 shows the variation of the relative unit costs of the block (over one) in relation to its size. To calculate these costs, the cost of the materials has been taken into account including the three main components of a block: laterals, manifold and control valve costs. The results presented correspond to a sprinkler irrigation set with laterals of $180 \mathrm{~m}$ fed from an intermediate point separated by $18 \mathrm{~m}$, and $1550 \mathrm{l} / \mathrm{h}$ sprinklers every $18 \mathrm{~m}$. Likewise, the cost of the manifold pipe has been counted with a variable diameter according to the size of the block, and the head valve. The contribution of each of the components (lateral, manifold and valve) has been broken down for each block area.

Figure 2 clearly shows that, in the case analysed, there is an optimum block area of 0.65 ha at which the unit cost is minimised, thus achieving a $30 \%$ reduction of cost in comparison with the most expensive solution. Analysing the contribution of each item shows that the contribution of the laterals is constant, as the increase in cost grows linearly with area. The valve has a decreasing weight as its relative value decreases as the area increases. However, from 0.81 ha upwards, an increase is observed owing to the increase in the size of the valve 
and thus, its price. The contribution of the manifold rises as, when the area increases, so does the manifold diameter and thus its unit cost. The conclusion is that the decisive factor for establishing the optimum block area is the valve, as when a larger size has to be chosen, this means an important increase in unit cost. These conclusions can be extrapolated for any type of pressurised irrigation system.

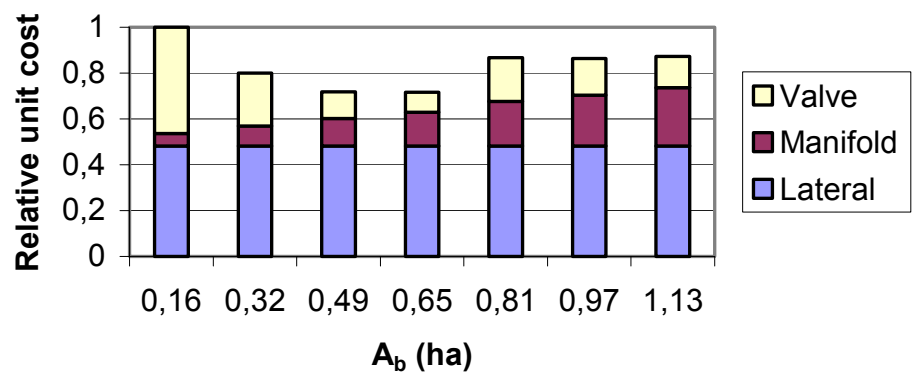

Figure 2: Variation of the relative unit cost of a block depending on its size for a sprinkler irrigation set.

The value of the optimum area will vary depending on the characteristics of the planned irrigation set. Thus it is advisable to calculate this for each specific case.

There are other costs not considered in this analysis, these include labour and trench excavation etc., which vary linearly with the area, thus their effect on the unit cost is constant. Another factor is the cost of the main and submain pipelines and control station, which will be higher the bigger the block is, which also supports the conclusion that there is an optimum block area with an intermediate value.

From the above, the number of blocks of a plot $\left(n_{b}\right)$ can be calculated by rounding up the quotient $A_{p} / A_{b o p t}$, and then,

$$
A_{b}=A_{p} / n_{b}
$$

The subdivision of a plot into blocks has a limit that derives from the availability of time between two consecutive irrigations (irrigation interval). Thus, it is important to calculate the maximum number of blocks $\left(n_{b \max }\right)$, which can be calculated as:

$$
n_{b \max }=\left[\frac{J_{r}}{t_{r g}}\right]_{w} \quad\left[I_{r} r_{p}\right]_{w}
$$

where:

$J_{r}$, operating time, number of hours of irrigation per day. interval.

$r_{p}$, operating ratio, quotient between operating days and the irrigation 
If in eqn (1), we find $t_{r g}$ and substitute it in (5) then:

$$
n_{b \max }=\left[\frac{q_{r g} J_{r} r_{p}}{q_{f b} 24}\right]_{w}
$$

Equation (6) is easier to apply than equation (5) as two variables, $t_{r g}$ and $I_{r}$, whose estimation involves a certain difficulty, have been eliminated. Moreover, the result must still be a whole number. Equation 6 shows the factors that allow $n_{b \max }$ to be increased and those that make it decrease.

Considering the definition of $q_{r g}$ (eqn (1)) and the partition on blocks (eqn (4), the supply flow in the plot will be:

$$
d=q_{r g} A_{b}
$$

The value resulting from equation (7) is significantly lower than the value of $d_{\max }$ mentioned above (eqn 2).

For very large plots, it may be that $n_{b}>n_{b \max }$, which is not possible owing to the limitations on the time available between irrigations mentioned above. Thus, if we adopt $n_{b}=n_{b \max }$, we will obtain values of $A_{b}>A_{b}$ opt which is possible hydraulically although it is not economically optimum. There is also the possibility of dividing a block into subunits (a subunit is understood as the lateral and manifold set fed by a pressure reduction valve), which may reduce the costs.

The results of the application of eqn (7) shows that the discharge allocation varies linearly with the area of the plot. There is a step when the number of blocks is increased, until the maximum number of blocks is reached. Then a constant slope is maintained. A drawback of this result is the wide variety of discharges obtained in depending on the area of the plot.

An alternative is that the plots between $A_{b \text { opt }}$ and $n_{b \text { max }} A_{b \text { opt }}$ have the possibility of making $A_{b \text { opt }}$ blocks. Applying these criteria would give figure 3 , a much simpler application in work, but which supposes a certain oversizing of the network capacity. Continuing this idea of simplifying, the plots between 0 and $A_{b o p t}$, could also be assigned $q_{r g} A_{b o p t}$.

The above methodology is applied below to the case developed by Clément and Galand [2]. The starting data is: $q_{f b}=0.6 \mathrm{l} \mathrm{s}^{-1} \mathrm{ha}^{-1}$, irrigation operating time $16 \mathrm{~h}, 8$ days of irrigation every $10, r_{p}=0.8$, irrigation specific discharge $=24.11$ $\mathrm{s}^{-1} \mathrm{ha}^{-1}$, optimum block area 0.5 ha.

Substituting in equation $6, \mathrm{n}_{\mathrm{b} \text { max }}=21$.

Figure 4 shows that the "proposed" assigned discharges for small plots are higher than those envisaged by Clément and Galand and, in contrast, for large plots the "proposed" assigned discharges are lower.

The reason for these differences can be observed in figures 5 and 6 . As mentioned above, Clément and Galand's method for small plots (fig 5) requires small blocks and thus a high number of blocks, which in principal, is not suitable for plots with fixed irrigation sets as it complicates the system. 


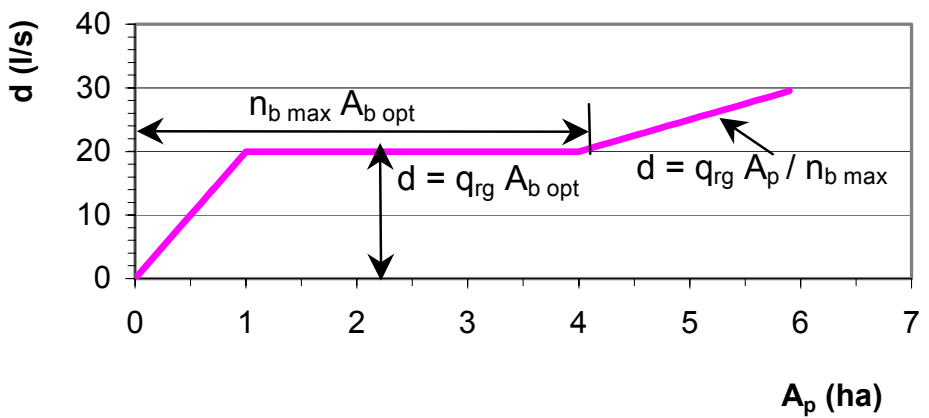

Figure 3: Allocation of discharge by plot area $\left(q_{r g}=201 \mathrm{~s}^{-1} \mathrm{ha}^{-1}, A_{b}\right.$ opt $=$ 1 ha, $\left.n_{b \max }=4\right)$.

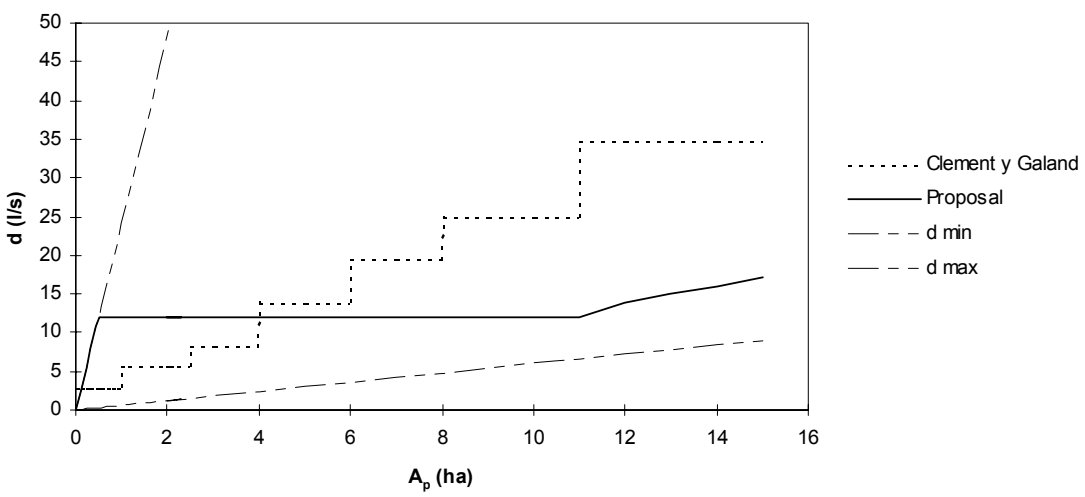

Figure 4: Comparison of the methodology proposed with that of Clément and Galand.

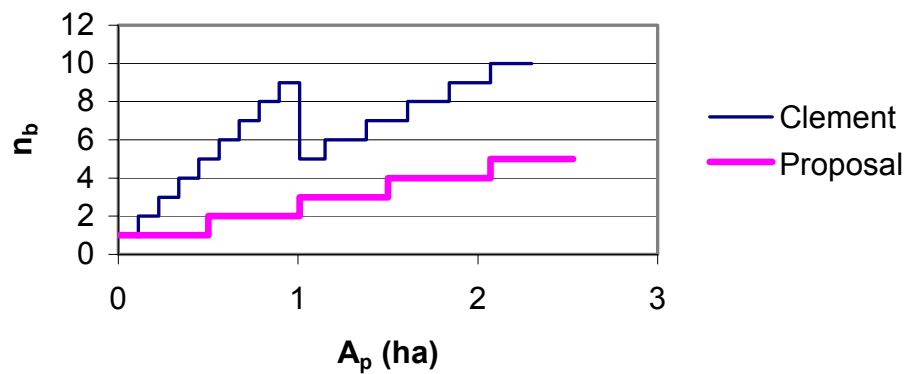

Figure 5: Number of blocks depending on plot size, for plots between 0 and 3 ha. 
In contrast, for large plots (fig 6) Clément and Galand's method forces blocks to be created that are much larger than the economic size. This is illogical bearing in mind that the number of blocks can be increased up to 21 for the case studied.

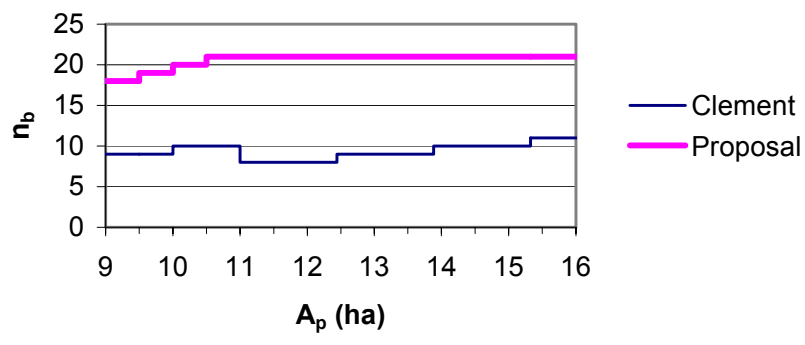

Figure 6: Number of blocks depending on plot size, for plots between 9 and 16 ha.

\section{Conclusions}

Clément and Galand's method for allocating flow to plots has the drawback of not being adjusted to the determining factors of fixed pressurised irrigation systems. For example, with this method, the block size depends on the area of the plot, while, as shown in this study, the economically optimum area of the block for a determined type of irrigation system has a specific fixed value. Another illogical result of this method is that the number of blocks for small plots is higher than for large plots.

Another fact is that nowadays, hydraulic valve technology with a flow limiter allows hydrants to be adjusted for any flow thus it is no longer necessary to establish standardised flows for hydrants as in Clément and Galand's method.

In the proposed method, the existence of a minimum cost block size is shown and which must be calculated for the main type of system that is envisaged. Thus, the flow to be supplied is that necessary for the planned irrigation method and the optimum area of the block. This results in the flow remaining constant independently of the size of the plot, as this is divided into blocks. When the maximum number of blocks is exceeded, the flow varies linearly with the area of the plot.

\section{Notation}

$A_{b}=$ Area of the block (ha)

$A_{\text {bopt }}=$ economic optimum size of the block (ha)

$A_{p}=$ Area of the plot, (ha)

$d_{\text {min }}=$ minimum supply flow $\left(1 \mathrm{~s}^{-1}\right)$

$d_{\text {max }}=$ maximum supply flow $\left(1 \mathrm{~s}^{-1}\right)$

$I_{r}=$ irrigation interval (days) 
$J_{r}=$ operating time per day $(\mathrm{h})$

$n_{b}=$ number of blocks

$n_{b \max }=$ maximum number of blocks

$q_{f b}=$ continuous gross specific discharge $\left(1 \mathrm{~s}^{-1} \mathrm{ha}^{-1}\right)$

$q_{p}=$ supply specific discharge $\left(1 \mathrm{~s}^{-1} \mathrm{ha}^{-1}\right)$

$q_{r g}=$ irrigation specific discharge $\left(l \mathrm{~s}^{-1} \mathrm{ha}^{-1}\right)$

$r_{p}=$ operating ratio

$t_{r g}=$ application time $(\mathrm{h})$

$t_{p}=$ irrigation time in the plot (h)

\section{References}

[1] Clément, R. Calcul des debits dans les reseaux d'irrigation fonctionant a la demande. La Houille Blanche, n.5. 1966.

[2] Clément R., Galand A. Irrigation par aspersion et reseaux collectifs de distribution sous pression. Eyrolles Editeur, Paris. 1979.

[3] Monserrat, J. et al. Analysis of Clément's first formula for irrigation distribution networks. Journal of Irrigation and Drainage Eng. Vol. 130 n.2. 2004. 